\title{
Pain-related quality of life related to mental health and sociodemographic indicators in adolescents
}

\author{
Perl Han Lee $1,2^{\star}$, Yi-Chun Yeh1,2*, Ray C. HsiaO³, Cheng-Fang Yen 1,2, Huei-Fan Hu 4 \\ ${ }^{1}$ Department of Psychiatry, Kaohsiung Medical University Hospital, Kaohsiung, Taiwan. \\ 2 Department of Psychiatry, School of Medicine, and Graduate Institute of Medicine, College of Medicine, Kaohsiung Medical University, Kaohsiung, Taiwan \\ ${ }^{3}$ Department of Psychiatry and Behavioral Sciences, University of Washington School of Medicine, and Department of Psychiatry, Children's Hospital, Seattle, WA. \\ ${ }_{4}^{4}$ Department of Psychiatry, Tainan Municipal Hospital, Tainan, Taiwan. \\ ${ }^{*}$ Dr. P-H Lee and Dr. Y-C Yeh contributed equally to this study.
}

Received: 11/28/2016 - Accepted: 5/4/2017

DOl: 10.1590/0101-60830000000122

\begin{abstract}
Background: A total of 6150 junior and senior high school students aged 11-18 years participated in this study. Their level of pain-related QOL was assessed using the pain subscale of the Taiwanese QOL Questionnaire for Adolescents. The severities of depression, suicidality, anxiety, and alcohol abuse were also examined. Objectives: This study examined the associations between pain-related quality of life (QOL) and sociodemographic characteristics and those between pain-related QOL and mental health problems such as depression, suicidality, anxiety, and alcohol abuse. Methods: The association of sociodemographic factors with pain-related QOL was examined through multiple regression analysis. The association of pain-related QOL with mental health problems was examined through logistic regression analysis. Results: The boys exhibited a higher level of satisfaction with pain-related QOL than did the girls. Older age was significantly associated with a lower level of satisfaction with pain-related QOL. Residential background, parental marital status, and parental education levels were not significantly associated with the level of satisfaction with pain-related QOL. Increased risks of depression, suicidality, anxiety, and alcohol abuse were significantly associated with a low level of satisfaction with pain-related QOL. Discussion: A low level of satisfaction with pain-related QOL is significantly associated with the risk of poor mental health. Adolescents with the correlates of low satisfaction with pain-related QOL should be monitored for the risk of mental health problems.
\end{abstract}

Lee PH et al. / Arch Clin Psychiatry. 2017;44(3):67-72

Keywords: Quality of life, pain, mental health, adolescent.

\section{Introduction}

Mental health problems are prevalent in adults with chronic nonmalignant pain ${ }^{1}$. Headache and joint, back, and neck pain increase the risks of depression-anxiety spectrum disorders and the chronic depression-anxiety course in adults ${ }^{2,3}$. Chronic nonmalignant pain, such as back pain and headache, is prevalent among adolescents ${ }^{4}$. However, to the best of our knowledge, few studies have examined the association between pain and mental health problems in adolescents.

An examination of the correlates of pain and their association with mental health problems in adolescents is warranted for the following reasons. First, adolescents undergo considerable biological changes with puberty ${ }^{5}$, and fast-growing muscles and bones may increase the likelihood of experiencing pain ${ }^{6}$. Second, high-technology devices and systems, such as computers, the Internet, and smartphones, have become important in the lives of adolescents ${ }^{7}$. The use of these high-technology devices and systems may reduce adolescents' engagement in physical exercise and increase their sedentary time, thus increasing their risk of pain ${ }^{8}$. Third, the construction of an emancipated identity is a major developmental task of adolescence ${ }^{5}$; therefore, pain and mental health problems developed during this stage may affect a person's identity in the long term. Hence, it is crucial to investigate pain and its association with mental health problems in adolescents.

Quality of life (QOL) is defined as people's perceptions of their own positions in life in the context of culture and value systems in which they live ${ }^{9}$. QOL is a multidimensional concept comprising psychological, physical, and social well-being ${ }^{10}$. Few studies have examined pain-related QOL and its sociodemographic correlates in adolescents. A previous study found that pain intensity is associated with a relatively poor functional status and poor psychological and somatic functioning in adolescents aged 12-18 years; pain-related QOL and the effect of pain on the families of the adolescents remained unchanged during the 3-year follow-up period in that study ${ }^{11}$. Additional studies should survey the sociodemographic correlates of pain-related QOL in adolescents to provide a reference for developing prevention and intervention strategies.

Although many studies have examined the association of malignant pain with depression and anxiety in adolescents in clinical units $^{12,13}$, to the best of our knowledge, few studies have examined the association between nonmalignant pain and mental health problems in adolescents in communities. Pain, depression, and anxiety may exhibit bidirectional relationships. Although a recent study investigating Norwegian adolescents determined that depression symptoms increased the risk of neck and shoulder pain ${ }^{14}$, another study investigating adolescents with cancer found that pain increased the risk of depression and anxiety ${ }^{15}$. Additional studies are necessary to investigate the association between nonmalignant pain and mental health problems in non-referred adolescents in communities.

A previous study reported a significant association between pain and suicidality in adults 3 ; the lifetime prevalence of suicide attempts in people with pain is approximately $5 \%-14 \%$, and the prevalence of suicidal ideation is approximately $20 \%{ }^{3}$. Chronic nonmalignant pain, such as migraines, increases the risk of suicidal attempts ${ }^{16}$. Other medical conditions, such as human immunoviral infection and multiple sclerosis, are also associated with suicidality ${ }^{17,18}$. A survey of the members of a chronic nonmalignant pain self-help organization revealed that the proportion of members considering suicide at the time of the survey was as high as $50 \%{ }^{19}$. However, most studies have not specifically focused on adolescents.

Address for correspondence: Cheng-Fang Yen. Department of Psychiatry, Kaohsiung Medical University Hospital. No. 100, Tzyou 1st Rd, Kaohsiung 807, Taiwan. Telephone: 886-7-3121101 ext. 6816. Fax: 886-7-3134761. E-mail: chfaye@cc.kmu.edu.tw. Huei-Fan Hu, Department of Psychiatry, Tainan Municipal Hospital, 670 Chongde Road, East District, Tainan 701, Taiwan. Telephone: 886-6-2609926. Fax: 886-6-2606351. E-mail: cych07205@gmail.com 
Researchers have also investigated the association between pain and alcohol use. Moderate alcohol consumption, as defined by The United States Department of Health and Human Services (2010), is associated with positive pain-related outcomes (e.g., higher QOL), whereas excessive consumption is associated with negative pain-related outcomes (e.g., higher pain severity) ${ }^{20}$. Zale et al. proposed that alcohol consumption can relieve acute pain, but pain can motivate alcohol consumption ${ }^{20}$. In addition, pain is a significant predictor of heavy drinking lapses during treatment ${ }^{21}$. Most studies have not focused on the association between pain and alcohol consumption in adolescents, except for a recent study that reported that chronic pain may not increase the risk of alcohol use in adolescents 22 .

The present study examined the sociodemographic correlates of pain-related QOL in adolescents and the correlation of painrelated QOL with depression, suicidality, anxiety, and alcohol abuse. Examining adolescents' pain-related QOL may provide information on their perception of pain and its effects on their daily lives. We hypothesized that differences exist in pain-related QOL among adolescents with various sociodemographic factors. We also hypothesized that pain-related QOL is significantly associated with depression, suicidality, anxiety, and alcohol abuse in adolescents.

\section{Methods}

\section{Participants}

Participants in this study were recruited from the 2009 Project for the Health of Children and Adolescents in Southern Taiwan, a research program examining the mental health status of children and adolescents living in four counties and three metropolitan areas in southern Taiwan ${ }^{23}$. In 2009, 254,130 students were studying in 205 junior high schools and 202,883 students in 143 senior high/vocational schools in this area. A stratified random sampling strategy was developed on the basis of the definitions of urban and rural districts in the Taiwan-Fukien Demographic Fact Book and school and grade characteristics and applied to ensure proportional representation of districts, schools, and grades. Five junior high schools and five senior high/vocational schools were randomly selected from urban districts. Similarly, five junior high schools and four senior high/vocational schools were randomly selected from rural districts. Furthermore, classes in these schools were stratified into three levels on the basis of grade: primary, junior high, and senior high/vocational school. Subsequently, 6703 high school students in grades 7-12 were randomly selected on the basis of the ratio of students in each grade. This study was approved by the Institutional Review Board of Kaohsiung Medical University.

\section{Instruments}

\section{Pain subscale of the Taiwanese OOL Questionnaire for Adolescents}

We used the self-reported pain subscale of the Taiwanese QOL Questionnaire for Adolescents (TQOLQA) to measure the levels of pain-related QOL in participants ${ }^{24}$. The pain subscale of the TQOLQA comprises three items: (1) "Do you worry about pain or discomfort?" (2) "Do you have any difficulty in managing or coping with pain or discomfort?" and (3) "Does your pain or discomfort interfere with things you need to do?" This instrument focused on both aspects of well-being and functionality. After the conversion of raw scores for reverse questions, a higher total subscale score indicates improved pain-related QOL over the preceding 2 weeks. The subscale is then standardized to range from 0 (lowest level of functioning) to 100 (highest level). In the present study, Cronbach's a coefficient of the pain subscale was 0.74 .

\section{Sociodemographic characteristics}

Data on characteristics such as age, sex, residential background (urban or rural), and paternal and maternal education levels were collected. In Taiwan, the duration of compulsory fundamental education is 9 years. Therefore, participants were categorized as those with parents with a high level of education (father or mother completed 9 years of compulsory fundamental education) and those with parents with a low level of education (father or mother did not complete 9 years of compulsory fundamental education).

\section{Mandarin Chinese version of the Center for Epidemiological Studies-Depression Scale}

The Mandarin Chinese version of the Center for Epidemiological Studies-Depression Scale (MC-CES-D) is a 20 -item selfadministered questionnaire that entails using a 4-point evaluation scale to assess the frequency of depressive symptoms in the preceding week ${ }^{25,26}$. Higher MC-CES-D scores indicate higher depression severity. In the present study, Cronbach's a coefficient of the MC-CES-D was 0.92. Moreover, participants whose total MC-CES-D score was 1 standard deviation (SD) higher than the mean score of the study population were classified as the group with significant depressive symptoms.

\section{Suicidality}

The 5-item questionnaire derived from the epidemiological version of the Kiddie Schedule for Affective Disorders and Schizophrenia ${ }^{27}$ was used to assess the occurrence of suicide attempts and four forms of suicidal ideation in the preceding year ${ }^{28}$. Each question elicited a "yes" or "no" response. Cohen's kappa coefficient of agreement $(\kappa)$ between participants' self-reported suicide attempts and their parents' reports was $0.541(p<0.001)^{28}$. Participants who responded "yes" to any of the five items were classified as having suicidal ideation or attempts.

\section{Taiwanese version of the Multidimensional Anxiety Scale for Children}

The Taiwanese version of the Multidimensional Anxiety Scale for Children (MASC-T) consists of 39 items that are answered on a 4-point Likert scale to evaluate participants' self-reported general anxiety symptoms ${ }^{29,30}$. A higher total MASC-T score represents a more severe level of general anxiety. Participants whose total MASC-T score was 1 SD higher than the mean score of the study population were classified as the group with significant general anxiety symptoms.

\section{CRAFFT Alcohol Abuse Screening Test}

The self-reported CRAFFT Alcohol Abuse Screening Test was developed to assess problematic alcohol use in adolescents ${ }^{31,32}$. A previous study ${ }^{31}$ demonstrated that a cutoff score of 2 on the CRAFFT Alcohol Abuse Screening Test can be used to identify adolescents with alcohol use disorder or problematic alcohol use on the basis of the Diagnostic and Statistical Manual of Mental Disorders ${ }^{33}$. In this study, we classified adolescents with a score of 2 or more on the CRAFFT Alcohol Abuse Screening Test as those having "problematic alcohol use".

\section{Statistical analysis}

A total of 6,445 students (96.2\%) agreed to participate in this study. Of the 258 students who did not participate in this study, 68 did not participate because of parental objections, 128 returned blank questionnaires, and 62 were absent when research assistants 
visited their classes. We recruited five research assistants with educational backgrounds of nursing, social work, or psychology. All research assistants received training to ascertain consistency and accuracy in the process of performing data collection of selfreported questionnaires. Research assistants explained the purpose and procedure of this study in each classroom during school hours and invited students to complete the research questionnaire anonymously. Participants could ask research assistants to answer them if they had questions regarding the contents of the research questionnaire. Participants who changed their mind could return blank questionnaires along with those from other students. All students received a gift worth NT\$33 (US\$1) at the end of the assessment.

Data analysis was performed using SPSS statistical software, version 18.0 (SPSS Inc., Chicago, IL, USA). The association of sociodemographic factors with pain-related QOL was examined in the two-step analysis. First, the association of sociodemographic factors with pain-related QOL was examined using Pearson's $r$ correlation and $t$ test. Second, factors that were significantly associated with pain-related QOL were further incorporated (independent variables) into a multiple linear regression analysis to examine their association with pain-related QOL (dependent variable). In addition, we used the standard criteria proposed by Baron and Kenny (1986) ${ }^{34}$ to examine whether the associations of some sociodemographic correlates with pain-related QOL were different in terms of other sociodemographic correlates. According to the criteria, moderation occurred when an interaction term for a predictor (sociodemographic correlate A) and a hypothesized moderator (sociodemographic correlate B) were significantly associated with a dependent variable (painrelated QOL) after controlling for the main effects of both predictors and hypothesized moderator variables. The associations of painrelated QOL (independent variable) with mental health problems (dependent variables) were examined using logistic regression analysis after controlling for the effects of sex and age. Two-tailed $p<.05$ was considered statistically significant.

\section{Results}

Table 1 lists the sociodemographic characteristics, level of painrelated QOL, and mental health indicators of the participants. Of the participants, 3216 were girls and 2934 were boys; their mean age was 14.8 years (SD: 1.8 years), and the mean score of painrelated QOL was 64.1 (SD: 17.2), ranging from 0 to 100 .

Table 2 shows the associations of sociodemographic factors with pain-related QOL examined using Pearson's r correlation and t test. Age was negatively associated with pain-related QOL $(p<.001)$. The boys had a higher level of satisfaction with painrelated QOL than did the girls $(p<.001)$. The adolescents with mothers with a high level of education reported a higher level of pain-related QOL than did those with mothers with a low level of education $(p=.048)$.

Age, sex, and maternal education level were further incorporated into multiple regression analysis to examine their association with pain-related QOL (Table 3). The condition index was 19.766, indicating the absence of a multicollinearity problem. The results of Model I indicated that male sex and old age were significantly associated with a lower level of satisfaction with pain-related QOL. Maternal education was not significantly associated with the level of satisfaction with pain-related QOL. Furthermore, we examined the moderating effect of sex on the association between age and painrelated QOL by including the interaction between sex and age in the regression analysis (Model II). However, the interaction between sex and age did not significantly moderate pain-related QOL.

Table 4 demonstrates the correlations of pain-related QOL with mental health problems. After the effects of age and sex were controlled for, a high level of satisfaction with pain-related QOL was significantly associated with reduced risks of depression, suicidality, anxiety, and alcohol abuse.
Table 1. Sociodemographic characteristics, the level of pain-related $00 \mathrm{~L}$, and mental health indicators ( $N=6150)$

\begin{tabular}{|l|c|l|l|}
\hline & $n(\%)$ & Mean (SD) & Range \\
\hline Sex & & & \\
\hline Girls & $3216(52.3)$ & & \\
\hline Boys & $2934(47.7)$ & & \\
\hline Age (years) & & $14.8(1.8)$ & $11-18$ \\
\hline Residential background & & & \\
\hline Urban & $3146(51.2)$ & & \\
\hline Rural & $3004(48.8)$ & & \\
\hline Parental marriage & & & \\
\hline Widowed, separated, and divorced & $919(14.9)$ & & \\
\hline Married and not separated & $5231(85.1)$ & & \\
\hline Paternal education & & & \\
\hline High & $4853(78.9)$ & & \\
\hline Low & $1297(21.1)$ & & \\
\hline Maternal education & & & \\
\hline High & $4796(78.0)$ & & \\
\hline Low & $1354(22.0)$ & & \\
\hline Pain-related Q0L & & $64.1(17.2)$ & $0-100$ \\
\hline Mental health indicators & & & \\
\hline Depression & $630(11.0)^{\mathrm{a}}$ & & \\
\hline Suicidality & $1977(32.3)^{\mathrm{b}}$ & & \\
\hline Anxiety & $739(13.9)^{\mathrm{c}}$ & & \\
\hline Alcohol abuse & $811(13.2)^{\mathrm{d}}$ & & \\
\hline
\end{tabular}

a: $n=5708 ; \mathrm{b} n=6127 ; c n=5327 ; \mathrm{d} n=6126$

Table 2. Association of sociodemographic factors with pain-related $\mathrm{QOL}$

\begin{tabular}{|l|c|c|c|c|}
\hline \multirow{2}{*}{} & \multicolumn{4}{|c|}{ Pain-related 00L } \\
\cline { 2 - 5 } & Pearson's $r$ & Mean (SD) & $t$ & $p$ \\
\hline Age & -0.094 & & & $<0.001$ \\
\hline Sex & & & & \\
\hline Girls & & $61.8(16.3)$ & -11.066 & $<0.001$ \\
\hline Boys & & $66.6(17.8)$ & & \\
\hline Residential background & & & & \\
\hline Urban & & $64.0(17.3)$ & -0.404 & 0.686 \\
\hline Rural & & $64.1(17.4)$ & & \\
\hline Parental marriage & & & & \\
\hline $\begin{array}{l}\text { Widowed, separated, and } \\
\text { divorced }\end{array}$ & & $63.8(17.3)$ & -0.431 & 0.666 \\
\hline Married and not separated & & $64.1(17.2)$ & & \\
\hline Paternal education level & & & & \\
\hline High & & $64.2(17.3)$ & 1.504 & 0.133 \\
\hline Low & & $63.4(16.8)$ & & \\
\hline Maternal education level & & & & \\
\hline High & & $64.3(17.4)$ & 1.974 & 0.048 \\
\hline Low & & $63.2(16.6)$ & & \\
\hline
\end{tabular}

Table 3. Association of sociodemographic factors with pain-related $\mathrm{QOL}$ : multiple regression analysis

\begin{tabular}{|l|c|c|c|c|c|c|}
\hline \multirow{2}{*}{} & \multicolumn{3}{|c|}{ Model I } & \multicolumn{3}{c|}{ Model II } \\
\cline { 2 - 8 } & Beta & $t$ & $p$ & Beta & $t$ & $p$ \\
\hline Sexa & 0.134 & 10.654 & $<0.001$ & 0.284 & 2.748 & 0.006 \\
\hline Age & -0.088 & -6.970 & $<0.001$ & -0.070 & -4.053 & $<0.001$ \\
\hline Maternal education levelb & -0.021 & -1.640 & 0.101 & -0.021 & -1.679 & 0.093 \\
\hline Sex $x$ Age & & & & -0.151 & -1.460 & 0.144 \\
\hline R square & \multicolumn{3}{|c|}{0.028} & \multicolumn{3}{c|}{0.028} \\
\hline
\end{tabular}

a: 0: girls; 1: boys; b: 0: high; 1: low. 
Table 4. Association of pain-related $\mathrm{OOL}$ with mental health problems: logistic regression analysis

\begin{tabular}{|c|c|c|c|c|c|c|c|c|c|c|c|c|c|c|c|c|}
\hline & \multicolumn{4}{|c|}{ Depression } & \multicolumn{4}{|c|}{ Suicidality } & \multicolumn{4}{|c|}{ Anxiety } & \multicolumn{4}{|c|}{ Alcohol abuse } \\
\hline & Wals & $p$ & OR & $95 \% \mathrm{Cl}$ & Wals & $p$ & $\mathrm{OR}$ & $95 \% \mathrm{Cl}$ & Wals & $p$ & $\mathrm{OR}$ & $95 \% \mathrm{Cl}$ & Wals & $p$ & $\mathrm{OR}$ & $95 \% \mathrm{Cl}$ \\
\hline $\begin{array}{l}\text { Pain- } \\
\text { related } \\
\mathrm{QOL}\end{array}$ & 295.026 & $<0.001$ & 0.957 & $0.952-0.962$ & 235.615 & $<0.001$ & 0.974 & $0.971-0.977$ & 421.331 & $<0.001$ & 0.946 & $0.941-0.951$ & 5.963 & 0.015 & 0.995 & $0.990-0.999$ \\
\hline Sex & 18.193 & $<0.001$ & \begin{tabular}{|l|}
0.678 \\
\end{tabular} & $0.568-0.811$ & 22.015 & $<0.001$ & 0.766 & $0.686-0.856$ & 63.546 & $<0.001$ & 0.485 & $\mid 0.406-0.579$ & 9.711 & 0.002 & 1.269 & $1.092-1.474$ \\
\hline Age & 3.448 & 0.063 & 1.046 & \begin{tabular}{|c|}
$0.997-1.098$ \\
\end{tabular} & 0.004 & 0.950 & 0.999 & $0.969-1.030$ & 26.886 & $<0.001$ & \begin{tabular}{|l|}
0.884 \\
\end{tabular} & $0.844-0.926$ & 24.010 & $<0.001$ & 1.108 & 1.064-1.155 \\
\hline
\end{tabular}

QOL: Quality of life.

\section{Discussion}

The results of the present study showed that the boys had a higher level of satisfaction with pain-related QOL than did the girls. Previous studies have also reported sex differences in pain experience ${ }^{35,36}$. Compared with men, women usually reported more frequent, severe, and persisting pain ${ }^{36}$. A study reported differences in central pain processing, including the magnitudes and locations of $\mu$-opioid system activation, between men and women ${ }^{37}$. Men have higher activation in the right dorsolateral prefrontal cortex, insula, and dorsal pons, whereas women have higher activation in the ventromedial prefrontal cortex, right anterior cingulate cortex, and left amygdala ${ }^{37}$. Additional studies should investigate whether these sex differences in central pain processing account for the sex difference in pain-related QOL found in this study. Moreover, hormonal changes during the menstrual cycle in women may partially account for the sex difference in pain-related QOL, because the sex difference in pain is mainly observed during reproductive years and is observed to a lesser extent in children or older adults ${ }^{38}$. In women, the various phases of the menstrual cycle may exert different effects on pain. A study reported that the luteal phase of the menstrual cycle is related to higher pain sensitivity, and higher rates of back pain, temporomandibular joint pain, and migraines occur at the end of the luteal phase ${ }^{36}$. Another study indicated that the reproductive status of women may influence the overall function of central opioid systems ${ }^{39}$.

Our study results revealed that older age (from the range of 11-18 years) was significantly associated with a lower level of satisfaction with pain-related QOL. Adolescents may encounter more physical conflicts and accidents as they become older; thus, they may also experience more pain. Cognitive and behavioral reactions to pain may also partially account for the association between age and pain-related QOL. Piira et al. reported that compared with younger children and adolescents, older children were more likely to use internalizing or catastrophizing coping strategies for pain ${ }^{40}$. Ineffective coping strategies for pain may further compromise their pain-related QOL. However, Myers et al. found that older adolescents may have higher pain tolerance than do younger adolescents ${ }^{41}$. Compared with younger adolescents, older adolescents may have higher self-confidence and are more well-equipped with appropriate skills to cope with pain. Additional studies should examine the association between age and pain-related QOL.

The results of this study indicated that a high level of satisfaction with pain-related QOL was significantly associated with a decreased risk of depression. Although many studies have explored the association between pain and depression ${ }^{42,43}$, it is difficult to establish the precise direction of causation. At the brain circuit level, mesolimbic dopamine system dysregulation has been proposed to be associated with both pain and depression ${ }^{44}$. At the physiological level, short-term stress activates dopaminergic neurotransmission in the ventral tegmental area and nucleus accumbens and drives motivation to cope with stress ${ }^{43,45}$. By contrast, chronic stress causes sustained activation and ultimately dysregulates the mesolimbic dopamine system ${ }^{46}$. These observations may partially account for the association between pain and depression. Although this crosssectional study did not determine the causal relationship between pain-related QOL and depression, we speculate that a high level of satisfaction with pain-related QOL indicates less susceptibility and more flexibility in the mesolimbic dopamine system and is hence associated with a decreased risk of depression.

In the present study, a high level of satisfaction with pain-related QOL was significantly associated with a decreased risk of suicidality. Previous studies have reported that chronic pain increased the risk of suicidality in adolescents ${ }^{47,48}$, even after controlling for depression ${ }^{49}$. At the brain circuit level, both pain and self-injurious acts are associated with the altered function of the endogenous opioid system, particularly $\beta$-endorphin and enkephalin neurotransmitters ${ }^{50}$. Chronic pain may result in endogenous opioid system dysfunction, and self-injurious acts may increase its functions ${ }^{51}$. Thus, we can reasonably speculate that a high level of satisfaction with painrelated QOL may reduce the need for endogenous opioid actions and is hence associated with a decreased risk of suicidality. Moreover, Cohen's kappa coefficient of agreement between participants' selfreported suicide attempts and their parents' reports evaluated using the 5-item questionnaire derived from the epidemiological version of the Kiddie Schedule for Affective Disorders and Schizophrenia was 0.541 , indicating a moderate agreement ${ }^{52}$. Detecting suicidal ideation of adolescents is a clinical challenge. Information from multiple sources, including adolescents themselves, families, peers, teachers, and social workers, is necessary for clinicians to evaluate the risk of suicidality among adolescents.

We observed that a high level of satisfaction with pain-related QOL was significantly associated with a decreased risk of anxiety. Although several studies ${ }^{53,54}$ have explored pain and anxiety in adolescents, extremely few studies have focused on pain-related QOL. A study reported that higher anxiety sensitivity correlates with a higher fear of pain, which is associated with increased pain disability ${ }^{5}$. Another study indicated that pain is related to functioning at a low, but not high, level of anxiety ${ }^{56}$. This finding provides an alternative perspective that anxiety may moderate the relationship between pain and QOL.

The present study found that a high level of satisfaction with pain-related QOL was significantly associated with a decreased risk of alcohol abuse. A previous study reported a curvilinear association between alcohol consumption and pain ${ }^{20}$. Moderate alcohol consumption was associated with positive pain-related outcomes, such as higher QOL, whereas excessive consumption was associated with negative pain-related outcomes, such as higher pain severity ${ }^{20}$. Another study showed that alcohol dependence and chronic pain share common neural circuits; therefore, pain sensitivity and alcohol use may exert reciprocal effects ${ }^{57}$.

Potential prevention strategies related to mental health problems are available. Clinicians should be more alert and pay more attention to psychiatric comorbidities when treating patients with pain. Awareness-raising activities and community psychoeducation are crucial to deliver accurate information regarding pain and mental health problems to the public. The incorporation of related psychoeducation into school programs can be more effective considering our targeted group of adolescents.

This study has several limitations. First, the cross-sectional research design of this study limited our ability to draw conclusions regarding the causal relationship between pain-related QOL and 
mental health problems examined in this study. Second, data in this study were exclusively self-reported. The use of one data source may have influenced our findings and resulted in shared-method variance. In addition, we did not specify the types of pain measured, which can be essential in further data analysis. Moreover, pain and discomfort are different aspects with different implications for prevention, assessment, and treatment; however, the design of questions in the questionnaire did not differentiate them. Third, although we examined the sociodemographic correlates of pain-related QOL, we did not examine the relationship of other factors, such as domestic violence or school bullying, with pain-related QOL. Therefore, taking into account of the limitations, additional studies should be conducted in the future not only to examine the relationship of these factors but also to explore the reasons underlying the association of pain-related QOL with depression, suicidality, anxiety, and alcohol abuse.

\section{Conclusion}

Sex and age are significantly associated with pain-related QOL. Thus, prevention and intervention programs for pain problems in adolescents should consider sex and age. A low level of satisfaction with pain-related QOL is significantly associated with increased risks of depression, suicidality, anxiety, and alcohol abuse, indicating that pain-related QOL is a clinical issue that deserves routine survey among adolescents.

\section{Funding}

This study was partially supported by grant NSC 97-2410-H-037003-SSS awarded by the National Science Council, Taiwan (ROC).

\section{Disclosure}

None declared.

\section{Ethical approval}

This study was approved by the Institutional Review Board of Kaohsiung Medical University.

\section{References}

1. Verhaak PF, Kerssens JJ, Dekker J, Sorbi MJ, Bensing JM. Prevalence of chronic benign pain disorder among adults: a review of the literature. Pain. 1998;77:231-9.

2. Gerrits MM, Vogelzangs N, van Oppen P, van Marwijk HW, van der Horst H, Penninx BW. Impact of pain on the course of depressive and anxiety disorders. Pain. 2012;153:429-36.

3. Tsang A, Von Korff M, Lee S, Alonso J, Karam E, Angermeyer MC, et al. Common chronic pain conditions in developed and developing countries: gender and age differences and comorbidity with depression-anxiety disorders. J Pain. 2008;9:883-91.

4. Richardson LP, Russo JE, Katon W, McCarty CA, DeVries A, Edlund MJ, et al. Mental health disorders and long-term opioid use among adolescents and young adults with chronic pain. J Adolesc Health. 2012;50:553-8.

5. Gemelli R. Normal child and adolescent development. Washington, DC: American Psychiatric Press; 1996.

6. Moon R. Sleep: What Every Parent Needs to Know. Elk Grove Village, IL: American Academy of Pediatrics; 2013.

7. Madden M, Lenhart A, Duggan M, Cortesi S, Gasser U. Teens and Technology 2013. Pew Research Center; 2013.

8. Hakala PT, Rimpela AH, Saarni LA, Salminen JJ. Frequent computerrelated activities increase the risk of neck-shoulder and low back pain in adolescents. Eur J Public Health. 2006;16:536-41.

9. Development of the World Health Organization WHOQOL-BREF quality of life assessment. Psychol Med. 1998;28:551-8.

10. Fuh J-L, Wang S-J, Lu S-R, Juang K-D. Assessing quality of life for adolescents in Taiwan. Psychiatry Clin Neurosci. 2005;59:11-8.

11. Hunfeld JA, Perquin CW, Bertina W, Hazebroek-Kampschreur AA, van Suijlekom-Smit LW, Koes BW, et al. Stability of pain parameters and pain-related quality of life in adolescents with persistent pain: a threeyear follow-up. Clin J Pain. 2002;18:99-106.

12. Hedstrom M, Ljungman G, von Essen L. Perceptions of distress among adolescents recently diagnosed with cancer. J Pediatr Hematol Oncol. 2005;27:15-22.

13. Hinds PS, Quargnenti AG, Wentz TJ. Measuring symptom distress in adolescents with cancer. J Pediatr Oncol Nurs. 1992; 9:84-6.

14. Myrtveit SM, Sivertsen B, Skogen JC, Frostholm L, Stormark KM, Hysing M. Adolescent neck and shoulder pain--the association with depression, physical activity, screen-based activities, and use of health care services. J Adolesc Health. 2014;55:366-72.

15. Bennett DS. Depression among children with chronic medical problems: a meta-analysis. J Pediatr Psychol. 1994;19:149-69.

16. Breslau N, Davis GC, Andreski P. Migraine, psychiatric disorders, and suicide attempts: an epidemiologic study of young adults. Psychiatry Res. 1991;37:11-23.

17. Stenager E, Knudsen L, Jensen K. Acute and chronic pain syndromes in multiple sclerosis. Acta Neurol Scand. 1991;84:197-200.

18. Breitbart W. Suicide risk and pain in cancer and AIDS patients. Current and Emerging Issues in Cancer Pain: Research and Practice. New York: Raven Press; 1993. p. 49-65.

19. Hitchcock LS, Ferrell BR, McCaffery M. The experience of chronic nonmalignant pain. J Pain Symptom Manage. 1994;9:312-8.

20. Zale EL, Maisto SA, Ditre JW. Interrelations between pain and alcohol: An integrative review. Clin Psychol Rev. 2015;37:57-71.

21. Witkiewitz K, Vowles KE, McCallion E, Frohe T, Kirouac M, Maisto SA. Pain as a predictor of heavy drinking and any drinking lapses in the COMBINE study and the UK Alcohol Treatment Trial. Addiction. 2015; 110:1262-71

22. Law EF, Bromberg MH, Noel M, Groenewald C, Murphy LK, Palermo TM. Alcohol and tobacco use in youth with and without chronic pain. J Pediatr Psychol. 2015;40:509-16.

23. Yen CF, Ko CH, Wu YY, Yen JY, Hsu FC, Yang P. Normative data on anxiety symptoms on the Multidimensional Anxiety Scale for Children in Taiwanese children and adolescents: differences in sex, age, and residence and comparison with an American sample. Child Psychiatry Hum Dev. 2010;41:614-23.

24. Fuh JL, Wang SJ, Lu SR, Juang KD. Assessing quality of life for adolescents in Taiwan. Psychiatry Clin Neurosci. 2005;59:11-8.

25. Chien CP, Cheng TA. Depression in Taiwan: epidemiological survey utilizing CES-D. Seishin Shinkeigaku Zasshi. 1985;87:335-8.

26. Radloff LS. The CES-D scale: A self-report depression scale for research in Rosenberg M. Society and the Adolescent Self-Image. New Jersey: Princeton University Press; 1977.

27. Puig-Antich J, Chambers W. The Schedule for Affective Disorders and Schizophrenia for School Age Children (Kiddie-SADS). New York: New York State Psychiatric Institute; 1978.

28. Tang T-C, Ko C-H, Yen J-Y, Lin H-C, Liu S-C, Huang C-F, et al. Suicide and Its Association with Individual, Family, Peer, and School Factors in an Adolescent Population in Southern Taiwan. Suicide Life Threat Behav. 2009;39(1):91-102.

29. March JS, Parker JDA, Sullivan K, Stallings P, Conners CK. The Multidimensional Anxiety Scale for Children (MASC): Factor Structure, Reliability, and Validity. J Am Acad Child Adolesc Psychiatry. 1997; 36:554-65.

30. Yen CF, Yang P, Wu YY, Hsu FC, Cheng CP. Factor structure, reliability and validity of the Taiwanese version of the Multidimensional Anxiety Scale for Children. Child Psychiatry Hum Dev. 2010;41:342-52.

31. Knight JR, Sherritt L, Harris SK, Gates EC, Chang G. Validity of brief alcohol screening tests among adolescents: a comparison of the AUDIT, POSIT, CAGE, and CRAFFT. Alcohol Clin Exp Res. 2003;27:67-73.

32. Ko CH, Yen JY, Yen CF, Chen CS, Weng CC, Chen CC. The association between Internet addiction and problematic alcohol use in adolescents: the problem behavior model. Cyberpsychol Behav. 2008;11:571-6.

33. Diagnostic and Statistical Manual of Mental Disorders (4th ed.). Washington, DC: American Psychiatric Association; 1994.

34. Baron RM, Kenny DA. The moderator-mediator variable distinction in social psychological research: Conceptual, strategic, and statistical considerations. J Pers Soc Psychol. 1986;51:1173-82.

35. Paller CJ, Campbell CM, Edwards RR, Dobs AS. Sex-based differences in pain perception and treatment. Pain Med. 2009;10:289-99.

36. Riley JL, 3rd, Robinson ME, Wise EA, Myers CD, Fillingim RB. Sex differences in the perception of noxious experimental stimuli: a metaanalysis. Pain. 1998;74:181-7. 
37. Naliboff BD, Berman S, Chang L, Derbyshire SW, Suyenobu B, Vogt BA, et al. Sex-related differences in IBS patients: central processing of visceral stimuli. Gastroenterology. 2003;124:1738-47.

38. Keogh E. Sex Differences in Pain. Rev Pain. 2008;2:4-7.

39. Zubieta JK, Dannals RF, Frost JJ. Gender and age influences on human brain mu-opioid receptor binding measured by PET. Am J Psychiatry. 1999;156:842-8.

40. Piira T, Taplin JE, Goodenough B, von Baeyer CL. Cognitive-behavioural predictors of children's tolerance of laboratory-induced pain: implications for clinical assessment and future directions. Behav Res Ther. 2002;40:571-84.

41. Myers CD, Tsao JC, Glover DA, Kim SC, Turk N, Zeltzer LK. Sex, gender, and age: contributions to laboratory pain responding in children and adolescents. J Pain. 2006;7:556-64.

42. Gerrits MM, van Marwijk HW, van Oppen P, van der Horst H, Penninx BW. Longitudinal association between pain, and depression and anxiety over four years. J Psychosom Res. 2015;78:64-70.

43. Horvitz JC. Dopamine gating of glutamatergic sensorimotor and incentive motivational input signals to the striatum. Behav Brain Res. 2002;137:65-74.

44. Finan PH, Smith MT. The comorbidity of insomnia, chronic pain, and depression: dopamine as a putative mechanism. Sleep Med Rev. 2013;17:173-83.

45. Abercrombie ED, Keefe KA, DiFrischia DS, Zigmond MJ. Differential effect of stress on in vivo dopamine release in striatum, nucleus accumbens, and medial frontal cortex. J Neurochem. 1989;52:1655-8.

46. Zautra AJ, Affleck GG, Tennen H, Reich JW, Davis MC. Dynamic approaches to emotions and stress in everyday life: Bolger and Zuckerman reloaded with positive as well as negative affects. J Pers. 2005;73:1511-38.
47. Wang SJ, Fuh JL, Juang KD, Lu SR. Migraine and suicidal ideation in adolescents aged 13 to 15 years. Neurology. 2009;72:1146-52.

48. Koenig J, Oelkers-Ax R, Parzer P, Haffner J, Brunner R, Resch F, et al. The association of self-injurious behaviour and suicide attempts with recurrent idiopathic pain in adolescents: evidence from a population-based study. Child Adolesc Psychiatry Ment Health. 2015;9:32.

49. van Tilburg MAL, Spence NJ, Whitehead WE, Bangdiwala S, Goldston DB. Chronic pain in adolescents is associated with suicidal thoughts and behaviors. J Pain. 2011;12:1032-9.

50. Nock MK. Self-injury. Annu Rev Clin Psychol. 2010;6:339-63.

51. Groschwitz R, Plener PL. The neurobiology of non-suicidal self-injury (NSSI): a review. Suicidol Online. 2012;3:24-32.

52. Altman DG. Practical Statistics for Medical Research. London England: Chapman and Hall; 1991.

53. von Gontard A, Moritz AM, Thome-Granz S, Equit M. Abdominal pain symptoms are associated with anxiety and depression in young children. Acta Paediatr. 2015;104:1156-63.

54. Tsao JC, Meldrum M, Kim SC, Zeltzer LK. Anxiety sensitivity and healthrelated quality of life in children with chronic pain. J Pain. 2007;8:814-23.

55. Martin AL, McGrath PA, Brown SC, Katz J. Anxiety sensitivity, fear of pain and pain-related disability in children and adolescents with chronic pain. Pain Res Manag. 2007;12:267-72.

56. Cohen LL, Vowles KE, Eccleston C. The impact of adolescent chronic pain on functioning: disentangling the complex role of anxiety. J Pain. 2010;11:1039-46.

57. Apkarian AV, Neugebauer V, Koob G, Edwards S, Levine JD, Ferrari L, et al. Neural mechanisms of pain and alcohol dependence. Pharmacol Biochem Behav. 2013;112:34-41. 